\title{
Interactive Humanoid Robots for a Science Museum
}

\author{
Masahiro Shiomi $^{1,2}$ Takayuki Kanda ${ }^{2}$ Hiroshi Ishiguro ${ }^{1,2}$ Norihiro Hagita $^{2}$
}

\author{
1 Osaka University \\ Osaka 565-0871 \\ Japan \\ 2 ATR IRC Laboratories \\ Kyoto 619-0288 \\ Japan \\ m-shiomi@atr.jp kanda@atr.jp ishiguro@atr.jp hagita@atr.jp
}

\begin{abstract}
This paper reports on a field trial with interactive humanoid robots at a science museum where visitors are supposed to study and develop an interest in science. In the trial, each visitor wore an RFID tag while looking around the museum's exhibits. Information obtained from the RFID tags was used to direct the robots' interaction with the visitors. The robots autonomously interacted with visitors via gestures and utterances resembling the free play of children [1]. In addition, they performed exhibitguiding by moving around several exhibits and explaining the exhibits based on sensor information. The robots were highly evaluated by visitors during the two-month trial. Moreover, we conducted an experiment in the field trial to compare the detailed effects of exhibit-guiding and free-play interaction under three operating conditions. This revealed that the combination of the free-play interaction and exhibit-guiding positively affected visitors' experiences at the science museum.
\end{abstract}

\section{Categories and Subject Descriptors}

H.5.2 [Information Interfaces and Presentation]: User Interfaces - Interaction styles, I.2.9 [Artificial Intelligence]: Robotics

\section{General Terms}

Design, Experimentation, Human Factors

\section{Keywords}

Commutation robot, Field trial, Human-robot interaction, Science museum robot

\section{INTRODUCTION}

The development of robots is entering a new stage where the focus is placed on interaction with people in their daily environments. The concept of the communication robot is rapidly emerging. The communication robot will act as a peer providing mental, communicational, and physical support. Such interactive tasks are of importance for allowing robots to take a part in human society. Many robots have already been applied to various fields in daily environments. Table 1 gives an overview of the previous works. There are mainly two kinds of fields: closed and open. The difference between a closed and an open environment lies in the people who are interacting. In a closed environment, such as an elementary school or an office, robots interact with a limited group of people [2-5]. On the contrary, we chose to work in an open environment because we expect that many people, in a wide- range of ages, will interact with robots. In line with this prospect, we have been developing a science museum guide robot that we believe to be a promising application.

There is a double benefit in choosing a science museum as the experiment field. On the one hand, visitors are going to have the opportunity to interact with the robots and experience the advanced technologies by which they are made, which is the fundamental purpose of a science museum. Thus, we can easily deploy our research to a real environment.

On the other hand, in a science museum we are naturally targeting people who are interested in science and are unlikely to miss the chance to interact with our robots; thus this field is one of the best choices for collecting feedback and examining the interaction between people and the communication robot in various tasks. The need for extensive and accurate feedback goes back to our belief that interaction with humans through tasks is one of the communication robot's essential roles. This feedback is vital for developing the ability for the robots to act appropriately in a daily living environment.

Some robots have already been developed [7, 8] that include functions like robust navigation and direction-giving in an open environment. However, the interactions of those robots are of the master-slave type (giving commands to the robot), which is quite different from the peer-type interaction we are expecting, i.e., as humans, pet animals, and AIBO [2] are capable of. We are attempting to emphasize the importance of this type of interaction. People think of their peers as "equals" when communicating with them. Thus we believe that achieving such a peer-type interaction between humans and robots makes the communication between them more natural and human-like. We can also expect that such an interaction will reduce the psychological distance between humans and robots. Moreover, particularly for the visitors of the science museum, it will help to stimulate their interest in science.

Our approach to these field experiments is unique: the robots use environmental sensors to work in a complex, crowded environment that would otherwise make simple functions such as person identification very difficult. We covered the experimental field at a science museum with cameras and wireless tag readers (RFID). The robot retrieves its coordinates from these ubiquitous sensors. It is able to identify visitors and call them by their names by detecting their RFID tags. This approach enables the robots to provide more pertinent information in their interaction, such as recommendations based on the visitor's movement history. We are exploring the potential of communication robots in various fields with this approach. 
TABLE 1 Various field experiments with interactive robots

\begin{tabular}{|c|c|c|c|c|c|c|c|c|}
\hline \multirow[b]{2}{*}{ Environment } & \multirow[b]{2}{*}{ Location } & \multirow[b]{2}{*}{ Purpose } & \multicolumn{2}{|l|}{ People } & \multicolumn{2}{|l|}{ Function } & \multicolumn{2}{|l|}{ Interaction } \\
\hline & & & $\begin{array}{l}\text { Wide age } \\
\text { range }\end{array}$ & $\begin{array}{l}\text { With many } \\
\text { people }\end{array}$ & Navigation & $\begin{array}{l}\text { Person } \\
\text { identification }\end{array}$ & Master slave & Peer type \\
\hline \multirow{4}{*}{ Closed } & Home & Entertainment [2] & $\mathbf{0}$ & $\mathbf{x}$ & $\mathbf{x}$ & $\mathbf{x}$ & - & $\mathbf{0}$ \\
\hline & Hospital & Mental care [3] & o & $\mathbf{x}$ & $\mathbf{x}$ & $\mathbf{x}$ & - & o \\
\hline & School & Language education [4] & $\mathbf{x}$ & o & $\mathbf{x}$ & o & - & o \\
\hline & Office & Guiding [5] & $\mathbf{x}$ & o & $\mathbf{0}$ & $\mathbf{x}$ & o & - \\
\hline \multirow{3}{*}{ Open } & Lobby & Interaction [6] & o & o & $\mathbf{x}$ & o & - & o \\
\hline & \multirow{2}{*}{ Museum } & Guidance \& navigation $[7,8]$ & o & o & o & $\mathbf{x}$ & o & - \\
\hline & & Interaction \& guidance [This paper] & o & o & $\mathbf{0}$ & $\mathbf{0}$ & - & $\mathbf{0}$ \\
\hline
\end{tabular}

This article reports on an experiment in which a system using many ubiquitous sensors and humanoid robots -- Robovies -guided the visitors of a science museum. In this setting the Robovies interacted with the visitors and showed them around to exhibits according to information from ubiquitous sensors, such as the visitors' positions and movement histories. During the twomonth experiment, visitors enjoyed interacting with the robots and highly appreciated them.

\section{SYSTEM CONFIGURATION}

We used four humanoid robots for interaction with visitors in guidance and giving explanations. The robots behaved as follows:

- One robot served as a guide to the exhibits.

- Two stationary robots explained the exhibits.

- As visitors prepared to leave, one robot greeted them by name, asked them to return their RFID tags, and said goodbye.

In addition, we installed many sensors to record the movements and positions of visitors via their RFID tags on the fourth floor of the Osaka Science Museum. The interaction data between robots and visitors were recorded on a central database.

The following sections describe the details of the Osaka Science Museum environment, the humanoid robots, and the sensors.

\subsection{Science museum environment}

Seventy-five exhibits were positioned on the fourth floor of the Osaka Science Museum. Figure 1 shows a map of the fourth floor of the museum, around which people walk in a counterclockwise direction.

Typically, visitors go through the following steps:

1) If a visitor decides to register as part of our project, such personal data as name, birthday, and age (under 20 or not) is gathered at the reception desk (Fig. 1, point A). The system binds that data to the ID of an RFID tag and automatically produces a synthetic voice for the visitor's name. The visitor receives a tag at the reception desk.

2) When the visitor strolls around the fourth floor wearing the RFID tag, the RFID tag readers detect its signal and the system records the information at regular time intervals of about $1.5 \mathrm{sec}$.

3) Four robots are placed at positions B, C, and D on the fourth floor, as shown in Fig. 1. After finishing, visitors return their tags at the exit point (Fig. 1, point E).

\subsection{Humanoid robots}

\section{1) Robovie:}

Figure 2 shows "Robovie," an interactive humanoid robot
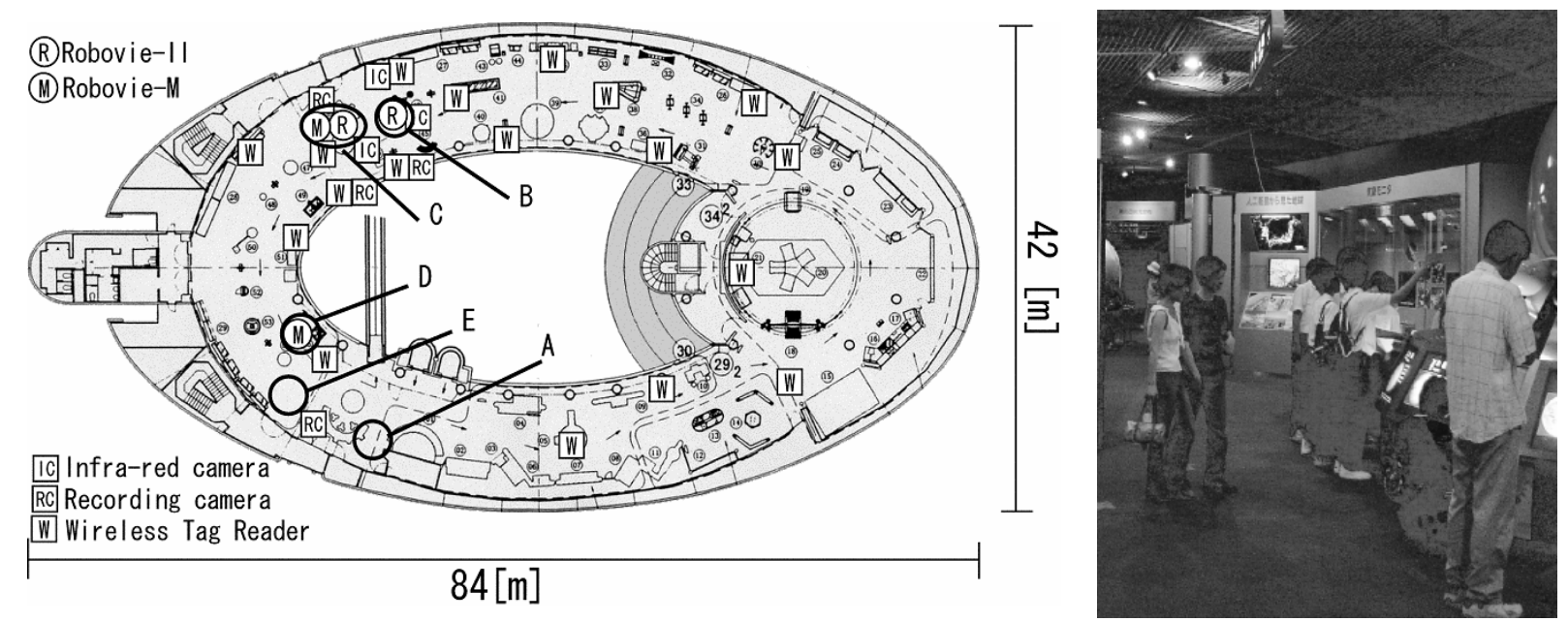

Figure 1. Map of the fourth floor of the Osaka Science Museum 
characterized by its human-like physical expressions and its various sensors. The reason we used humanoid robots is because a human-like body is useful to naturally control the attention of humans [9]. The human-like body consists of a head, a pair of eyes, and two arms. When combined, these parts can generate the complex body movements required for communication. We decided on a robot height of $120 \mathrm{~cm}$ to decrease the risk of scaring children. The diameter was $40 \mathrm{~cm}$. The robot has two $4 * 2$ DOFs (degrees of freedom) in its arms, 3 DOFs in its head, and a mobile platform. It can synthesize and produce a voice via a speaker. We also attached an RFID tag reader to Robovie [4] that enables it to identify the individuals around it. Two of the four robots used in this experiment were Robovies.

\section{2) Robovie-M:}

Figure 3 shows a "Robovie-M" humanoid robot characterized by its human-like physical expressions. We decided on a height of 29 $\mathrm{cm}$ for this robot. Robovie-M has 22 DOFs and can perform twolegged locomotion, bow its head, and do a handstand. We used a personal computer and a pair of speakers to enable it to speak, since it was originally unequipped for that.

The two other robots in this experiment were Robovie-Ms.

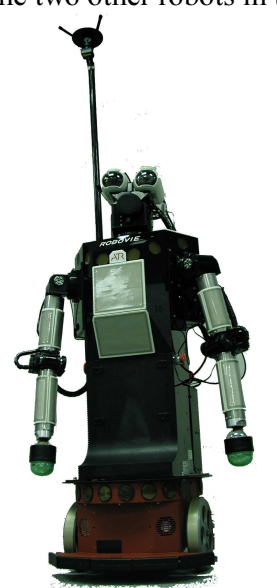

Figure 2. Robovie

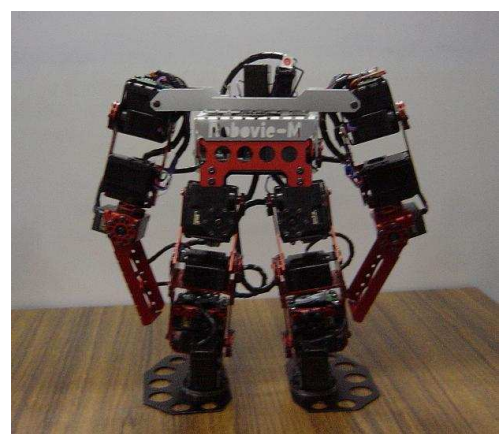

Figure 3. Robovie-M

\subsection{Embedded sensors in an environment:}

On the fourth floor of the Osaka Science Museum, we installed 20 RFID tag readers (Spider-IIIA, RF-CODE), which included the two equipped on the Robovies, three infrared sensors, and four video cameras. All sensor data were sent to a central server database through an Ethernet network.

In the following sections, we describe each type of sensor used.

1) RFID tag readers:

We used an active type of RFID tag. This technology enables easy identification of individuals: detection is unaffected by the occurrence of occlusions, the detection area is wide, and the distance between the tag reader and an RFID tag can be roughly estimated. Such benefits are suitable for large environments.

However, drawbacks include low accuracy over long distances and the inability to detect exact positions. We compensated for these shortcomings by installing many RFID tag readers in the environment.
To achieve approximate distance estimation, we set the RFID tag readers to have eight levels of sensitivity. Detection areas, however, are affected by the position of the RFID tag readers and reflections due to walls. Therefore, we measured each detection area prior to the experiment. We then attached the tag readers in positions two meters above the floor, and to successfully detect the tags we had to set the reader sensitivity level to at least five.

Fig 1 shows an example of the positioning of tag readers. We placed them around particular exhibits, so that the system could detect whether visitors approached them. Moreover, since a tag reader's detection field has a torus shape, the system can estimate the tag position by superposing the circles calculated from the reader outputs (Figure 5).

\section{2) Infrared cameras:}

We placed an infrared LED on top of a Robovie and attached infrared cameras to the ceiling to determine the robot's correct position. The system produces binary images from the infrared cameras and detects bright areas. It calculates absolute coordinates with a reference to the weighted center of the detection area and sends them to the database.

Infrared camera positions are shown in Fig. 1. The distance between the floor and the ceiling is about $4 \mathrm{~m}$. The width and height of images from an infrared camera is 320 and 240 pixels, respectively. One pixel represents about $1 \mathrm{~cm}^{2}$ of area.

\section{3) Video cameras:}

The video camera positions are also shown in Fig. 1. The output images of each video camera are recorded by a PC and used to analyze the data generated by the experiment.

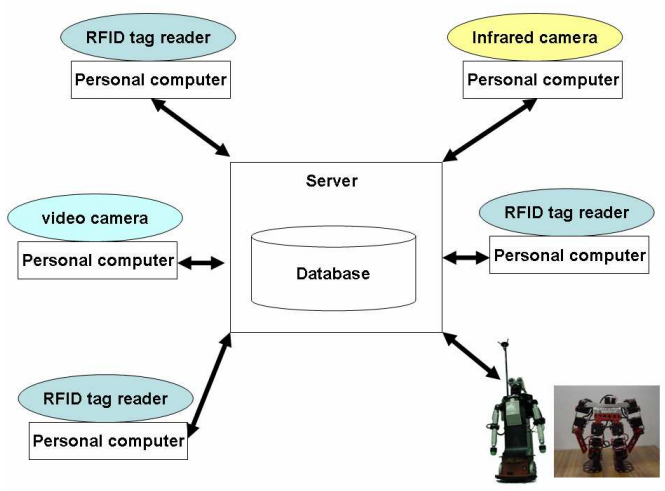

Figure 4. The ubiquitous sensors

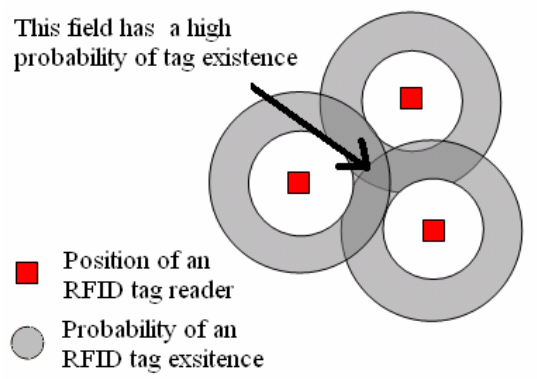

Figure 5. Detection fields of the RFID tag 


\section{ROBOT BEHAVIORS}

In this section, we introduce the roles and behaviors of the robots. For friendly interaction with visitors, robots need information about them. For example, children's interest increases when the machines call them by name [4]. Moreover, human interactions are characterized by a shared memory of events.

It is difficult for the robots themselves to acquire this information about visitors, such as their names and memories. However, sensors enable them to capture this data through, for example, an Ethernet network. That way the robots can act more intelligently and overcome the limitations of their features. In addition, in this system we use Robovies as sensors because they contain an RFID tag readers. In effect, they became not only interactive robots but also part of the sensor system.

\subsection{Locomotive robot}

We used a Robovie for the locomotive robot that moved around in parts of the environment, interacted with visitors, and guided them to exhibits.

\section{1) Interaction with humans: Childlike interaction}

The robot can engage in such childlike behavior as handshaking, hugging, and the game of "rock, paper, and scissors." Moreover, it has such reactive behaviors as avoidance and gazing at a touched part of its body, as well as such patient behavior as solitary playing and moving back and forth. Figure 6 shows interaction scenes between Robovies and visitors.

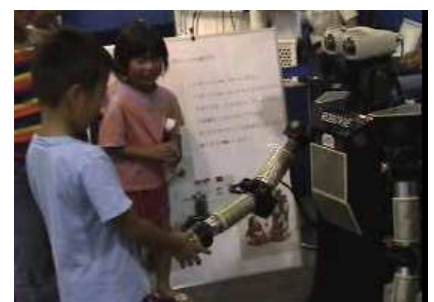

(a) Robovie shakes hands

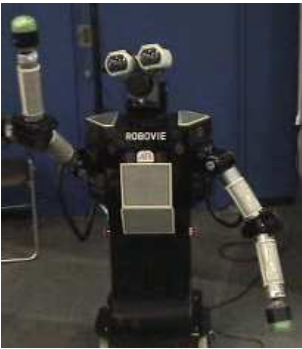

(c) Robovie saying goodbye

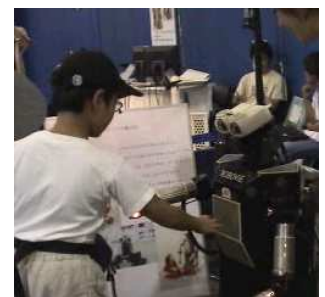

(b) A child touching Robovie

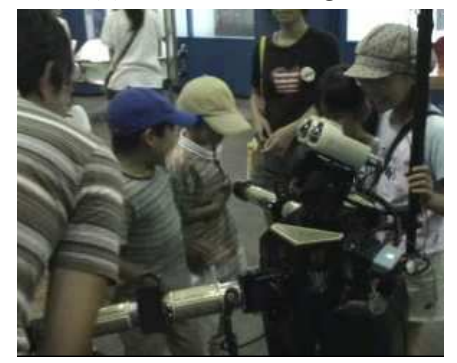

(d) Robovie hugging children
Figure 6. Scenes of interaction between visitors and Robovies

\section{2) Interaction with humans: Using information from RFID tags}

The robot can detect RFID tag signals around itself by using its RFID tag reader, which allows it to obtain personal data on visitors using RFID tag IDs. It can greet visitors by name or wish them a happy birthday, and so on. In addition, the system records the time that visitors spend on the fourth floor of the Osaka Science Museum. The robot can behave according to that time.
3) Guiding people to exhibits: Human guidance

The robot can guide people to four kinds of exhibits by randomly determining the target. Figure 7 shows an example of this behavior. When bringing visitors to the telescope, the robot says, "I am taking you to an exhibit, please follow me!" (a), and approaches the telescope $(b, c)$. It suggests that the person look through it and then talks about its inventor (d).

4) Guiding people to exhibits: Using information from RFID tags

The RFID tags' data are also used for interaction. We used the amount of time that visitors spent near an exhibit can use to judge whether visitors tried an exhibits. For example, when an RFIDtagged visitor has stayed around the "magnetic power" exhibit longer than a predefined time, the system assumes that the visitor has already tried it. Thus, the robot says, "Yamada-san, thank you for trying 'magnetic power.' What did you think of it?" If the system assumes that the visitor has not tried it, the robot will ask, "Yamada-san, you didn't try 'magnetic power.' It's really fun, why don't you give it a try?"

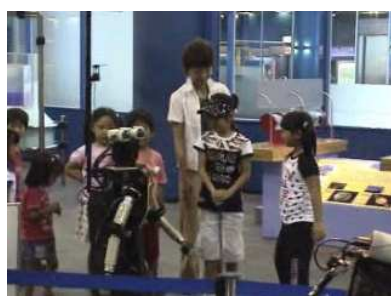

(a)

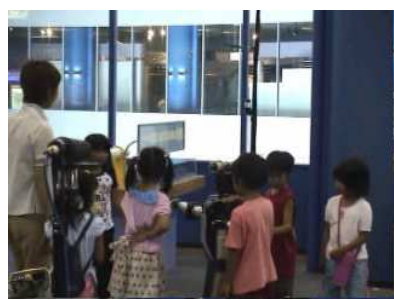

(c)

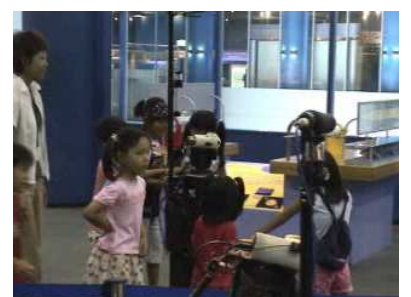

(b)

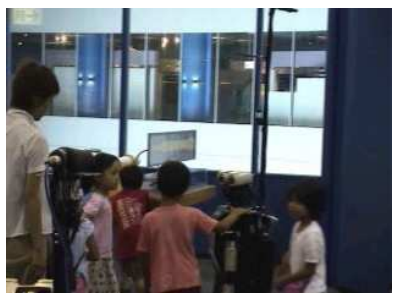

(d)
Figure 7. Robovie guiding visitors to the telescope

\subsection{Robots that talk with each other}

Two stationary robots (Robovie and Robovie-M) casually talk about the exhibits as humans do with accurate timing because they are synchronized with each other using an Ethernet network. The topic itself is intelligently determined by data from RFID tags. By knowing the previous visiting course of a visitor, the robots can try to interest the visitor in an exhibit he or she overlooked by starting a conversation on that exhibit.

Figure 8 shows robots talking. The flow and an example of dialogue are given below:

(1) Robovie-M explains an exhibit.

(2) Robovie asks Robovie-M a question about it. For example, "Who made it?"

(3) Robovie-M answers the question and expounds on its answer.

(Robovie-M): "That chair can float, even if a person is sitting on it." 
(Robovie): "That's incredible! How does it do that?'

(Robovie-M): "By magnetic power."

(Robovie): "I wonder if I can sit on that..."

(Robovie-M): "I doubt it."

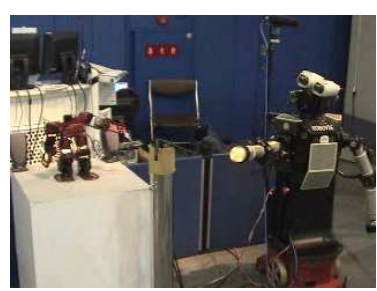

(a) Two robots talking

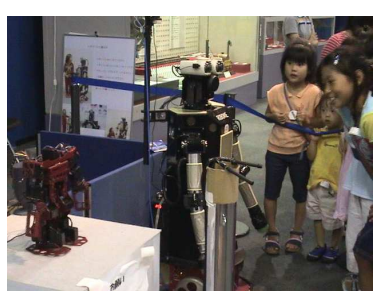

(b) Two robots talking to visitors
Figure 8. Scenes of robots talking to each other

\subsection{A robot bidding farewell}

This robot is positioned near the exit and, after requesting data from their RFID tags, says goodbye to the departing visitors. It also reorients visitors on the tour who are lost by examining the visitor's movement history and time spent on the fourth floor of the Osaka Science Museum, which was recorded by the system. If visitors walk clockwise, they will immediately see this robot at the beginning and will be pointed in the right direction by the robot. Figure 9 shows a scene with this robot.

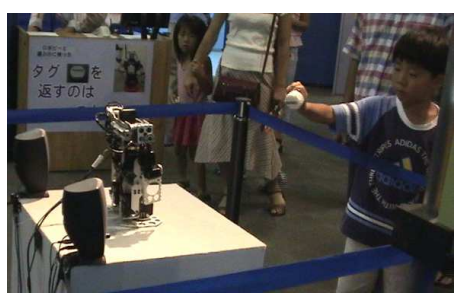

(a) The visitor talking to the robot

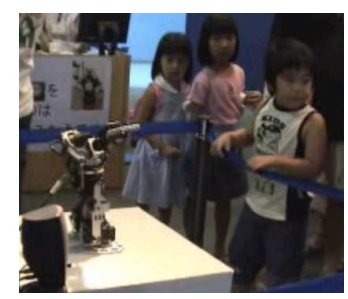

(b) The robot telling the way
Figure 9. The robot bidding farewell

\section{EXPERIMENT}

\subsection{A two-month exhibition}

We performed experiments to investigate the impressions made by robots on visitors to the fourth floor of the Osaka Science Museum during a two-month period. As they departed the fourth floor, we asked visitors to complete a questionnaire by ranking five factors on a scale of 1-to-5, where 5 is the most positive. They were also encouraged to give other opinions on the robots.

By the end of the two-month period, the number of visitors had reached 91,107, the number of subjects who wore RFID tags was 11,927 and the number of returned questionnaires was 2,891. Figure 10 shows the results. It indicates that most visitors had a good impression of the robots and they did not feel anxiety about robots in the future. Figure 11 and 12 display the age and gender of subjects who wore RFID tags and returned questionnaires. These results indicate that most questionnaires were returned by woman and visitors in their 30's and 40's. We think that this trend happened because only adults were asked to fill in the questionnaires, and the typical visitor group was a mother accompanying her child.) Most opinions were:
- We had a really good time.

- I had fun because the robots called me by name.

- We felt close to the robots.

The results revealed that visitors held favorable impressions toward the presence of the robots. Moreover, visitors described their favorite robot behavior, such as hugging, the calling out of names, and so on. Such behaviors are basic elements of human society.

The freely described opinions of visitors were analyzed and revealed that visitors' opinions of the robots differed according to age [10]. For example, younger respondents did not necessarily like the robots more than elder respondents.

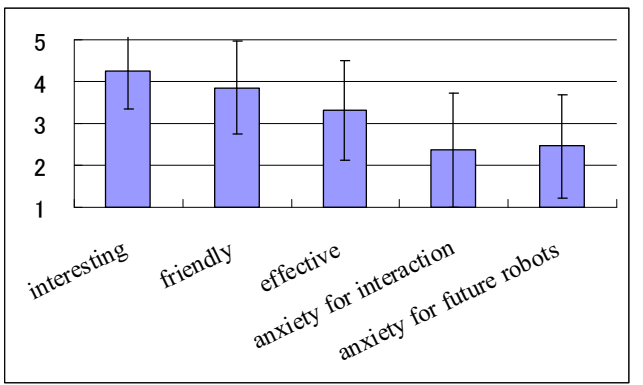

Figure 10. Results of returned questionnaires

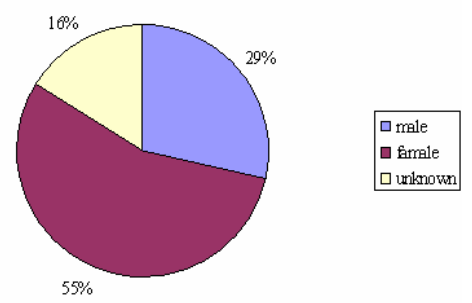

Figure 11. Gender of visitors

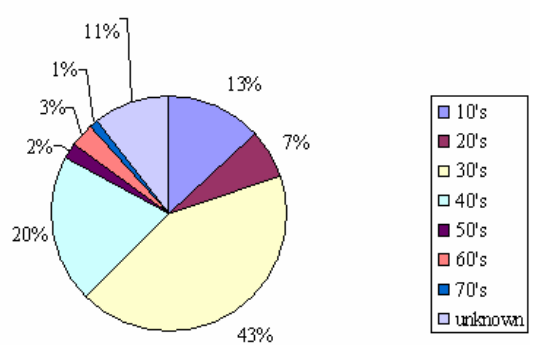

Figure 12. Age of visitors

\subsection{Experiments on the behavior of robots}

We performed experiments in which we examined the behavior of robots under three operating conditions during one week. We randomly exchanged conditions between the morning and the afternoon. The subjects were the visitors who had RFID tags and played with the robots. After their interaction ended, we asked them to fill out a questionnaire in which they rated three items on a scale of 1-to-7, where 7 is the most positive.

The items were "Presence of the robots" (What did you think about the presence of robots in the science museum?), "Usefulness as a guide" (What was the degree of the robots" 
usefulness for easily looking around the exhibits?), and "Experience of science \& technology" (How much did the robots increase your interest in science and technology?). The subjects were also encouraged to provide other opinions about the robots as well. The three operating conditions were the following:

\section{1) Interaction}

Robots behaved according to predefined functions. Each robot engaged in basic interaction, as described in Section 3.1.1. No guide function was performed

\section{2) Guidance}

The role of the robots was limited to guiding and giving explanations. Each robot only behaved as described in Section 3.1.3.

\section{3) Interaction, guidance and using RFID tags}

In this operating condition the robots not only combined the previous two operating conditions but also used data from the RFID tags. Each robot preformed every kind of behavior introduced in Section 3.1.

It is difficult to compare the conditions of "using RFID" and "not using RFID". For example, in the "Guide" condition, using information on the RFID tag necessitates that the robot behave interactively, such as calling someone by name. Also, it is difficult to compare the effects of each behavior in the crowded situations. Thus, we use this operating condition for comparing the importance of the information on the RFID tags between the above conditions.

\subsection{Results of robots' behavior}

About 100 questionnaires were returned for each operating condition. Figure 13 shows the results and their averages, which are mostly above 6 . There was a significant difference for the following item: "Experience of science \& technology," as to whether the robot was in the "Interaction, guidance and using RFID" operating condition or in another condition $(\mathrm{p}<.05)$.

A comparison of the three conditions' results based on analysis of variance revealed no significant differences between the two items of "Presence of the robots" and "Usefulness as a guide."

Concerning this last item, here are examples of some of the most remarkable feedback:

- Children developed an interest in other exhibits after being led to them or having them explained by a robot.

- Children were amused by the robot's reactions to being touched and became interested in new exhibited items when following it.

These opinions indicate that interest in science is developed by possible interaction with robots. Other feedback opinions attest to the good impressions that robots made on subjects.

On the other hand, robots sometimes could not interact well with visitors. For example, some children were afraid to interact with robots and some visitors did not care about the robots' actions. Moreover, visitors' opinions included some negative impressions such as "we couldn't talk to the robots because the speech ability of the robots was not good". These show that the interaction ability of the robots was not good enough for an open environment.

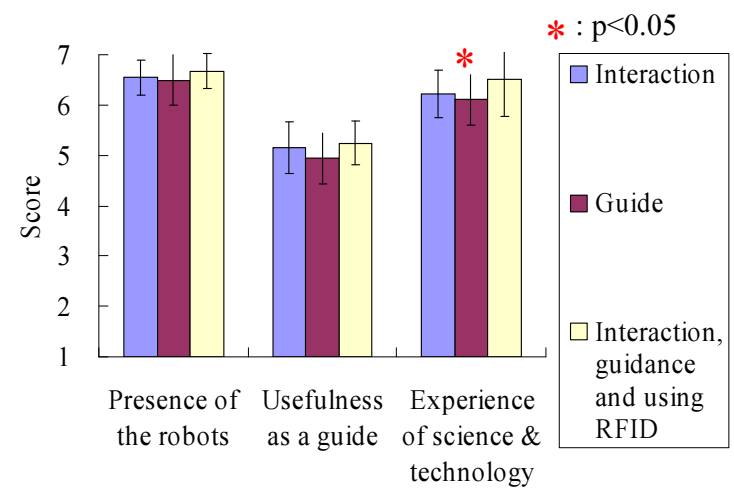

Figure 13. Results for the three operating conditions

\section{DISCUSSION}

\subsection{Contributions to HRI research}

More than ninety thousand people visited the exhibition, more than ten thousand people interacted with the robots and wore RFID tags, and about three thousand people returned questionnaires. The results showed that most visitors evaluated the robots highly.

In the "Interaction, guide, and using RFID" condition, "Experience of science \& technology" showed a significant difference. Human-guide-like guiding with childlike free-play interaction attracted the interest of visitors more than simply guiding.

We believe that free-play interaction and human-guide-like guiding improve the visitors' interest in science technology because the visitors played actively with the robots and with the exhibits via the interaction of the robots.

In addition to these findings, we believe that this trial demonstrated the positive possibility of using interactive robots in open environments, which is one of the most important contributions of this work. Human-robot interaction was considered only as a kind of entertainment when research on interactive robots started. Our perspective (and that of many other researchers) is very different. We believe that robots will be part of the fundamental infrastructure of our society and will support a wide range of our daily activities. We believe that it is important to demonstrate possible applications of interactive robots, such as those in $[4,6]$, and this work particularly demonstrated the possibility for use in an open environment where both the novelty and interactivity of the robots were appreciated.

\subsection{The system components}

Generally, it is not so easy to evaluate large-scale systems used in a field trial. It sometimes forces us to show easily-comparable results, such as the questionnaire answers, as the result of the field trial. However, it does not always demonstrate the essential meaning of the trial, particularly for a system in its earlier phase. In our research, many system components were integrated in the whole system, but the questionnaire answers, such as "most of the visitors were happy about the robots," do not tell us anything about how each system component contributed to the system and how we can further investigate human-robot interaction.

Here, we discuss how each of the system components contributed to the whole system, and how visitors interacted with 
them by introducing interesting scenes of visitors' interaction as a case study.

\section{Locomotive robot}

- Often there were many adults and children crowded around the robot. In crowded situations, mainly a few children simultaneously interacted with the robot in turn.

- Similar to Robovie's free-play interaction in a laboratory [1], children shook hands, played the paper-rock-scissors game, hugged, and so forth. Sometimes they imitated the robot's body movements, such as the robot's exercising.

- When the robot started to move to a different place (in front of an exhibit), some children followed the robot to the destination.

- After the robot explained about a telescope exhibit, one child went to use the telescope. When he came back around the robot, another child used the telescope.

- Its name-calling behavior attracted many visitors. They tried to show the RFID tags embedded in the nameplates to the robot. Often, when one visitor did this, several other visitors began showing the robots their nameplates, too, as if they were competing to have their names called.

- When the robot called a child's name, the child and his/her mother smiled and were satisfied with it, while other children again tried to show their nameplates. Such a scene was often observed. Sometimes, a child and his/her parent waited more than ten minutes to have their name called by the robot. (The robot called one of the visitors' names around the robot, which is highly affected by the radio strength. Some tags' radio strength seems to have been weaker than the others'.)

- A visitor reported that when the robot moved to him, he thought that it was aware of him, which made him happy.

We believe that there are many implications in these observations. In particular, it reminds us of the importance of making a robot move around. It enables the robot to attract people to interact with it. Moreover, as shown in the scene where children followed the locomotive robot, it drew their attention to the exhibit, although the exhibit (a telescope) was relatively not so attractive. (There are many attractive exhibits to move and operate to gain an understanding of science, such as a pulley and a lever.) We demonstrated that robots can provide visitors with the opportunity to play with and study science through exhibits they might have otherwise missed.

Another interesting aspect was that it might moderate an overly crowded situation where people gathered around the robot due to its novelty. As shown in the above scene, the robot's locomotion triggers people who are very interested in it to follow it while others may not.

\section{Robots that talk to each other}

- There were two types of typical visitors' behavior. One is just to listen to the robots' talk. For example, after listening to it, the visitors talked about the exhibit that was explained to them, and sometimes visited the exhibit.

- The other is to expect to have their name called. In this case, the visitors paid rather less attention to the robots' talk, and instead showed their name to the robots, which is similar to the actions observed around the locomotive robot. Often the visitor left the front of the robot just after his/her name was called.

One implication is that showing a robots' conversation can attract people and convey information to them, even though its interactivity is very low. Such examples are also shown in our other work $[11,12]$. Currently, robots' interactivity is actually quite low because of their limited sensing ability, particularly in a daily environment. This forces an interactive robot to use limited sensor information, such as only tactile sensors without speech recognition.

\section{Robot bidding farewell}

- There were two types of typical visitors' behavior. One is just to watch the robot's behavior.

- The other is, again, to expect to have their name called. In this case, the visitors often showed their name to the robots.

- Many children listened to the robot's utterances, such as "Yamada-kun, thank you for visiting today. Please return the nameplate to the exit booth over there." After that, they immediately returned the nameplates to the exit booth.

The cost of Robovie-M is far cheaper than that of Robovie-II. Although its functionality is very limited, such as its small size, no embedded speech functions (we placed a speaker nearby), and no sensors (an RFID reader was also placed nearby), it entertained many visitors. We believe that this indicates the effectiveness of ubiquitous sensors. The simple robot was endowed with ubiquitous sensors, giving it a more attractive interaction capability. Particularly, the effectiveness of the name-calling behavior was again demonstrated, as seen in the children's behavior of returning their RFID tags.

\section{RFID tags and tag readers}

As shown in the scenes of interaction with the robots, the application of RFID technology largely promoted the humanrobot interaction, particularly with regard to the name-calling behavior. However, the information obtained from the distributed RFID tag readers made a relatively small contribution to the system. Robots talked to the visitors about their exhibit-visiting experience, such as "You did not see the telescope exhibit, did you? It is very interesting. Please try it," based on the information from the RFID reader network, but it seemed to be less attractive and impressive to the visitors. This was also pointed out in our previous work [13]. (At that time, we thought it was due to the small environment.) Perhaps, robots are too novel for visitors, so they highly appreciate their experience of interacting with the robots while less attention is paid to the detailed services that they offer.

\section{Other ubiquitous sensors}

Regarding the ubiquitous sensors other than the RFID tags, their role was limited. The infrared camera supplied the exact position of the robot, which was very helpful in the crowded environment. We believe that there will be much information from ubiquitous sensors available for human-robot interaction, which should be included in our future work. (We conducted further research on this, which is under submission to this HRI conference [14]).

\subsection{Needs for social abilities in the robots}

Although we demonstrated our robots in an open field as an early application of interactive robots, our robots were basically designed to interact with a few people who are already interested in interacting with robots. Here, we discuss the need for social abilities in the robots for open environments, which our robots currently lack, by showing several scenes of interaction.

- When the robot started to explain about an exhibit, visitors were sometimes too busy to try to evoke reactions from the robot, 
such as by touching the robot, and failed to follow the context. This resulted in the failure of the explanation: visitors continued to play with the robot while it spoke about an exhibit, and it did not react to the visitors.

- The robot tried to draw the attention of a child by calling his name, "Play with me, Yamada-kun." At that time there were a crowd of visitors who wore nameplates. All of the others got interested in having their name called, such as by showing their nameplate to the robot, and no longer listened to the robot's utterances except for the name-calling behavior.

- Children found the robot's exercise behavior and hugging behavior entertaining; however, when there were only adults around the robot, these behaviors made them hesitate to interact with it. Interestingly, when a mother was in front of the robot with her children, she enjoyed its hugging behavior.

We believe that these scenes demonstrate the needs of further study and development of the robot's social abilities. The first scene reminds us of the importance of drawing people's attention to an appropriate target, such as the robot itself, or the target object it is talking about, depending on the situation. However, the second scene warns us against drawing an individual's attention in a crowded situation, although it is powerful to use one's name for that purpose. As shown in the second scene, although the name-calling behavior was attractive and powerful for drawing the attention of a visitor, it sometimes disturbed the social-attention of the visitors. In addition, from the third scene, we can learn the importance of paying careful attention to people's attributes, including but not limited to their adult-child and group membership. (We have implemented many minor issues on this, such as calling children's names prior to adults' in name-calling behavior but it seems not enough).

\section{CONCLUSION}

We have developed an interactive robot system that combines autonomous robots and ubiquitous sensors. The system guided visitors through a science museum with human-like interaction, such as calling their names in a free-play behavior and explaining exhibits with voice and gestures. In a two-month exhibition, 91,107 people visited the Osaka Science Museum, 11,927 of whom wore RFID tags to participate in the field trial. The results from questionnaires revealed that almost all visitors evaluated these robots highly. Furthermore, we investigated the influence of the free-play interaction and guidance of the robots. As a result, we found that the robots that performed childlike free-play interaction and guided visitors were the best in attracting attention to scientific explanations.

\section{ACKNOWLEDGMENT}

We wish to thank the staff at the Osaka Science Museum for their kind cooperation. We also wish to thank the following ATR members for their helpful suggestions and cooperation: Tatsuya Nomura, Hideaki Terauchi, Takugo Tasaki, Daniel Eaton, Toshihiko Shibata, Koutarou Hayashi, Masaaki Kakio, Taichi Tajika, and Fumitaka Yamaoka. This research was supported by the Ministry of Internal Affairs and Communications of Japan.

\section{REFERENCES}

[1] Ishiguro, H., Imai, M., Maeda, T., Kanda, T., and Nakatsu, R. Robovie: an interactive humanoid robot, Int. J. Industrial Robot, Vol. 28, No. 6, pp.498-503, 2001.

[2] Fujita, M. "AIBO: Toward the era of digital creatures," Int. J. Robot. Res., vol. 20, no. 10, pp. 781-794, 2001.

[3] Shibata, T. "An overview of human interactive robots for psychological enrichment", The proceedings of IEEE November 2004

[4] Kanda, T., Hirano, T., Eaton, D., and Ishiguro, H. "Interactive Robots as Social Partners and Peer Tutors for Children: A Field Trial," Journal of Human Computer Interaction, Vol. 19, No. 1-2, pp. 61-84, 2004.

[5] Asoh, H., Hayamizu, S., Hara, I., Motomura, Y., Akaho, S., and Matsui, T. "Socially Embedded Learning of the OfficeConversant Mobile Robot Jijo-2," Int. Joint Conf. on Artificial Intelligence (IJCAI), 1997.

[6] Gockley, R., Bruce, A., Forlizzi, L., Michalowski, M., Mundell, A., Rosenthal S., Sellner, B., Simmons, R., Snipes, K., Schultz, Alan C., and Wang. J. "Designing Robots for Long-Term Social Interaction," IROS2005. pp 2199-2204

[7] Burgard, W., Cremers, A. B, Fox, D., Hähnel, D., Lakemeyer, G., Schulz, D., Steiner, W., and Thrun, S. "The Interactive Museum Tour-Guide Robot," Proc. National Conference on Artificial Intelligence (AAAI), 1998.

[8] Siegwart, R., and et al. "Robox at Expo.02: A Large Scale Installation of Personal Robots". Robotics and Autonomous Systems, 42, 203-222, 2003

[9] Imai, M., Ono T., Ishiguro, H.. Physical Relation and Expression: Joint Attention for Human-Robot Interaction, Proceedings of the 10th IEEE International Workshop on Robot and Human Communication (RO-MAN2001), pp.512517, 2001

[10] Nomura, T., Tasaki, T., Kanda, T., Shiomi, M., Ishiguro, H., and Hagita, N. Questionnaire-Based Research on Opinions of Visitors for Communication Robots at an Exhibition in Japan, International Conference on Human-Computer Interaction (Interact 2005), 2005.

[11] Kanda, T., Ishiguro, H., Ono, T., Imai, M., and Mase, K. "Multi-robot Cooperation for Human-Robot Communication," IEEE International Workshop on Robot and Human Communication (ROMAN2002), pp.271-276, 2002.

[12] Hayashi, K., Kanda, T., Miyashita, T., Ishiguro, H., and Hagita, N. Robot Manzai - Robots' conversation as a passive social medium-, IEEE International Conference on Humanoid Robots (Humanoids2005), 2005.

[13] Koide, Y., Kanda, T., Sumi, Y., Kogure, K., and Ishiguro, H. An Approach to Integrating an Interactive Guide Robot with Ubiquitous Sensors, IROS2004, pp.2500-2505, 2004.

[14] Nabe, S., Kanda, T., Hiraki, K., Ishiguto, H. Kogure, K., Hagita, N.Analysis of Human Behavior in an Open Field to Improve Communication Robots, HRI2006,(to appear) 